\title{
A Unified Approach to Dual Gabor Windows
}

\author{
Tobias Werther, Ewa Matusiak, Yonina C. Eldar, Member, IEEE, and Nagesh K. Subbana
}

\begin{abstract}
In this paper, we describe a new method for studying the invertibility of Gabor frame operators. Our approach can be applied to both the continuous $\left(\right.$ on $\mathbb{R}^{d}$ ) and the finite discrete setting. In the latter case, we obtain algorithms for directly computing the inverse of Gabor frame-type matrices equivalent to those known in the literature. The framework we propose can also be used to derive other (known) results in Gabor theory in a unified way such as the Zibulski-Zeevi representation. The approach we suggest is based on an adequate splitting of the twisted convolution, which, in turn, provides another twisted convolution on a finite cyclic group. By analogy with the twisted convolution of finite discrete signals, we derive a mapping between the sequence space and a matrix algebra which preserves the algebraic structure. In this way, the invertibility problem reduces to the analysis of finite matrices whose entries are sequences supported on corresponding cosets. Using Cramer's rule and proving Wiener's lemma for this special class of matrices, we obtain an invertibility criterion that can be applied to a variety of different settings. This alternative approach provides further insight into Gabor frames, as well as a unified framework for Gabor analysis.
\end{abstract}

Index Terms-Gabor frame operator, Janssen coefficients, Zak transform, Zibulski-Zeevi representation.

\section{INTRODUCTION}

G ABOR analysis is a widely used signal processing method for decomposing and reconstructing signals from their time-frequency projections, that is, their sampled version of the short-time Fourier transform. Gabor representation is used in many applications ranging from speech processing and texture segmentation to pattern and object recognition, among others [1], [8], [11], [15].

One of the advantages of Gabor expansions is the highly structured system inherited from the uniform time-frequency lattice which allows for efficient time-frequency algorithms. A major part of Gabor analysis relies on frame theory, which deals with overcomplete representations [2], [3], [10]. When using an overcomplete Gabor system to decompose a signal, the reconstruction is no longer unique. The most popular choice is based on the canonical (minimal norm) dual Gabor frame [12], [13].

Manuscript received December 6, 2005; revised June 26, 2006. This work was supported in part by the Ollendorff Minerva Center and in part by the European Union's Human Potential Programme under contract HPRN-CT-2003-00285 (HASSIP). The work of Y. C. Eldar was supported in part by the Israel Science Foundation under Grant 536/04. The associate editor coordinating the review of this manuscript and approving it for publication was Dr. Zoran Cvetkovic.

T. Werther and E. Matusiak are with the Faculty of Mathematics, University of Vienna, 1090 Vienna, Austria (e-mail: tobias.werther@univie.ac.at; ewa.matusiak@univie.ac.at).

Y. C. Eldar and N. K. Subbana are with the Department of Electrical Engineering, The Technion-Israel Institute of Technology, 32000 Haifa, Israel (e-mail: yonina@ee.technion.ac.il; nagesh@techunix.technion.ac.il).

Color versions of one or more of the figures in this paper are available online at http://ieeexplore.ieee.org.

Digital Object Identifier 10.1109/TSP.2006.890908
Computing the canonical dual involves inverting the frame operator associated with the given Gabor frame. The frame operator has a lot of structure emerging from the commutativity with corresponding time-frequency shifts which is the consequence of the uniformity of the underlying time-frequency lattice. This structure gives rise to many important and well-known results on representing a frame operator. The representation we are concerned with is the so-called Janssen representation. Many results are known for Gabor systems to constitute a frame which implies invertibility [2], [7]. Standard algorithms make use of the highly structured Gabor frames in order to efficiently compute the canonical dual frame. Recently, in [14], the authors described an alternative way to compute other dual Gabor frames where they based their approach on the twisted convolution of the Janssen coefficients of the corresponding operators.

Here, we extend the idea of [14] by using a different strategy which allows far better insights into the problem. Specifically, we only deal with sequences and show explicitly how efficient inversion schemes can be derived by rather simple (though sophisticated) manipulations of the twisted convolution. The essential idea is to split up the twisted convolution into a finite number of sums that can be incorporated into a special matrix algebra.

Our framework yields further insights into the concept of inverting Gabor frame-type operators [defined in (5)]. Dealing only with the coefficient sequence of the Janssen representation without using other constructs of Gabor analysis allows for a simple and straightforward extension to higher dimensions. The approach works also for the finite-dimensional setting whereas this has not been the case in [14]. The rather elementary techniques in our method provide a better understanding of Gabor frame-type operators than the widely known Zibulski-Zeevi representation from a conceptual point of view. Moreover, the method we present is equivalent to the two mentioned above, in the sense that they are connected by a unitary transformation.

The paper is organized as follows. Section II is devoted to notation that will be used throughout the paper. In Section III, we state the main concepts of Gabor frame theory and show how the twisted convolution comes into play when dealing with inversion problems. Section IV is a short introduction to twisted convolution in the most simplest setting which serves the purpose of motivating our approach. In Section V, we describe the main theoretical results which leads to constructive methods for inverting Gabor frame-type operators. In Section VI, we outline the equivalence of our method to two other ideas that are described in the literature. Section VII is devoted to an analysis of our method in the finite 1-D signal space.

\section{NOTATION}

Central objects in time-frequency analysis are modulation and translation operators. For $x, \xi, t \in \mathbb{R}$, we define the transla- 
tion operator and the modulation operator on $L^{2}(\mathbb{R})$ by

$$
\begin{aligned}
T_{x} f(t) & =f(t-x) \\
M_{\xi} f(t) & =e^{2 \pi i \xi t} f(t)
\end{aligned}
$$

respectively. Many technical details in time-frequency analysis are linked to the commutation law of the translation and modulation operator, namely

$$
M_{\xi} T_{x}=e^{2 \pi i x \xi} T_{x} M_{\xi} .
$$

In Section III, we study twisted convolution of two finite length sequences $f=\left(f_{k, l}\right), g=\left(g_{k, l}\right)$ indexed by $\mathbb{Z}_{p} \times \mathbb{Z}_{p}$, where $\mathbb{Z}_{p}$, for any natural number $p$, will always denote a finite cyclic group, that is a set of elements $\{0, \ldots, p-1\}$ with addition modulo $p$. We think of $f$ and $g$ as vectors of length $p^{2}$, where the entries are in lexicograpic order, that is, for example for $p=2, f=\left(f_{0,0}, f_{0,1}, f_{1,0}, f_{1,1}\right)$. When studying such convolutions, the concept of block circulant matrices arises. A block circulant matrix of size $p^{2} \times p^{2}$ is a matrix of the form

$$
\mathcal{C}\left(G_{0}, G_{p-1}, \ldots, G_{1}\right):=\left(\begin{array}{cccc}
G_{0} & G_{p-1} & \cdots & G_{1} \\
G_{1} & G_{0} & \cdots & G_{2} \\
\vdots & \vdots & & \vdots \\
G_{p-1} & G_{p-2} & \cdots & G_{0}
\end{array}\right)
$$

where, in our setting, each block $G_{k}, k=0, \ldots, p-1$, is a $p \times p$ matrix.

In our derivations, we use two kinds of delta functions. The first, denoted by $\delta$, means a Dirac sequence in $\ell^{1}\left(\mathbb{Z}^{2}\right)$, that is a sequence with value 1 at $(0,0)$ and zeros elsewhere. The second, denoted by $\delta_{r, s}$ for $r, s \in \mathbb{Z}$, takes value 1 when $r=s$ and zeros elsewhere. Sometimes we write $\delta_{q \mathbb{Z}, r}$ for some $q \in \mathbb{Z}$, which means that it is 1 when $r \in q \mathbb{Z}$ and zeros elsewhere.

In Section VI and Appendix B and C, we use the symbol $\mathbb{F}$ to denote the Fourier transform operator, that is, for $h \in L^{2}(\mathbb{R})$, $\mathcal{F} h$ is the Fourier transform of $h$.

\section{UNIFIED APPROACH TO AlteRnATIVE DUAL GABOR FRAMES}

The fundamental idea of Gabor analysis is to build up a basic system out of time-frequency shifts of a single window function, where the shifts are usually taken according to a regular lattice in the time-frequency domain. For practical purposes, it is important to obtain a complete stable basis system which depends on the window function and the time-frequency lattice. In the case of Gabor expansions, this means that the Gabor system provides a frame. An excellent treatise on Gabor analysis is given in [7].

We now introduce the basic notation and concepts of Gabor frame theory. Let $g$ be in $L^{2}(\mathbb{R})$ and $\Lambda$ a separable lattice in $\mathbb{R}^{2}$ of the form

$$
\Lambda=\alpha \mathbb{Z} \times \beta \mathbb{Z}
$$

where $\alpha$ and $\beta$ are two positive numbers, called lattice constants, such that $\alpha \beta=p / q$ with $p, q \in \mathbb{Z}$ relatively prime. This corresponds to the rational case of Gabor frames [7]. The case $p=1$ is referred to as integer oversmpaling. A Gabor system $\mathcal{G}(g, \alpha, \beta)$ is defined by

$$
\mathcal{G}(g, \alpha, \beta)=\left\{M_{\beta m} T_{\alpha n} g \mid m, n \in \mathbb{Z}\right\} .
$$

We call $\mathcal{G}(g, \alpha, \beta)$ a frame if there exists two positive constants $A, B$ such that the inequality

$$
A\|f\|^{2} \leq \sum_{m, n \in \mathbb{Z}}\left|\left\langle f, M_{\beta m} T_{\alpha n} g\right\rangle\right|^{2} \leq B\|f\|^{2}
$$

holds for all $f \in L^{2}(\mathbb{R})$. In this case, the frame operator $S$, mapping $L^{2}(\mathbb{R})$ into $L^{2}(\mathbb{R})$, given by

$$
S f=\sum_{m, n \in \mathbb{Z}}\left\langle f, M_{\beta m} T_{\alpha n} g\right\rangle M_{\beta m} T_{\alpha n} g
$$

is positive and invertible. If $\mathcal{G}(g, \alpha, \beta)$ constitutes a frame, then there exists a so-called dual element

$$
\tilde{g}=S^{-1} g
$$

which induces the dual Gabor frame $\mathcal{G}(\tilde{g}, \alpha, \beta)$ enjoying the property that every $f \in L^{2}$ has the following time-frequency representation:

$$
\begin{aligned}
f & =\sum_{m, n \in \mathbb{Z}}\left\langle f, M_{\beta m} T_{\alpha n} \tilde{g}\right\rangle M_{\beta m} T_{\alpha n} g \\
& =\sum_{m, n \in \mathbb{Z}}\left\langle f, M_{\beta m} T_{\alpha n} g\right\rangle M_{\beta m} T_{\alpha n} \tilde{g} .
\end{aligned}
$$

The dual frame element $\tilde{g}$ is not unique. In general, there exist other window functions that give rise to (4). In [14], the authors present a method that allows us to efficiently compute alternative dual Gabor elements. Their approach is based on the concept of choosing two different Gabor frames, say $\mathcal{G}(g, \alpha, \beta)$ and $\mathcal{G}(\gamma, \alpha, \beta)$, such that the frame-type operator

$$
S_{g, \gamma} f \sum_{m, n \in \mathbb{Z}}\left\langle f, M_{\beta m} T_{\alpha n} g\right\rangle M_{\beta m} T_{\alpha n} \gamma
$$

is invertible. Then, the function $S_{g, \gamma}^{-1} \gamma$ induces a dual frame for $\mathcal{G}(g, \alpha, \beta)$. As shown in [14], this strategy may improve stability and gives rise to an alternative method for designing dual Gabor elements.

The central object in the approach described in [14] is the frame-type operator $S_{g, \gamma}$. In particular, the authors derive conditions under which, for given Gabor frames $\mathcal{G}(g, \alpha, \beta)$ and $\mathcal{G}(\gamma, \alpha, \beta), S_{g, \gamma}$ is invertible. There basically exist two (equivalent) methods to study the invertibility of $S_{g, \gamma}$.

The first constructive approach goes back to [16] in which the authors use a piecewise Zak transform to reduce the problem of invertibility to a finite matrix problem. We will describe this idea in Section VI.

The second method is essentially described in [14]. It is based on the Janssen representation of $S_{g, \gamma}$ [9], that is

$$
S_{g, \gamma}=\sum_{m, n \in \mathbb{Z}}\left\langle\gamma, M_{m / \alpha} T_{n / \beta} g\right\rangle M_{m / \alpha} T_{n / \beta} .
$$

We consider only those functions $g$ and $\gamma$ such that $\left\langle\gamma, M_{m / \alpha} T_{n / \beta g}\right\rangle, m, n \in \mathbb{Z}$, is an $\ell^{1}$ sequence. A class 
of functions with this property is the Feichtinger algebra $S_{0}$ [6] defined by

$$
S_{0}=\left\{f \in L^{2}\left|\|f\|_{S_{0}}:=\int_{\mathbb{R}^{2}}\right| V_{\psi} f(x, \xi) \mid d x d \xi<\infty\right\}
$$

where $V_{\psi} f(x, \xi)=\int_{\mathbb{R}} f(t) \overline{\psi(t-x)} e^{-2 \pi i \xi t} d t$ denotes the short-time Fourier transform with respect to the Gaussian window $\psi(x)=e^{-\pi x^{2}}$. Examples of functions in $S_{0}$ are the Gaussian and continuous B-splines of any order [6]. The Feichtinger algebra is an extremely useful space of test functions and of "good" window functions in the sense of time-frequency localization. Rigorous descriptions of $S_{0}$ can be found in [6] and references therein. This will guarantee that the sequence $a_{m, n}=\left(\left\langle\gamma, M_{m / \alpha} T_{n / \beta} g\right\rangle\right)$, that is a sampled short-time Fourier transform, is in $\ell^{1}\left(\mathbb{Z}^{2}\right)$, which implies absolute convergence of the Janssen representation.

Due to the Janssen representation, we can transfer the study of invertibility of frame-type operators to the study of the twisted convolution of $\ell^{1}$-sequences which is defined by

$$
(a \curvearrowleft b)_{m, n}=\sum_{k, l \in \mathbb{Z}} a_{k, l} b_{m-k, n-l} \omega^{(m-k) l}
$$

with $\omega=e^{-2 \pi i q / p}$ and $a, b \in \ell^{1}\left(\mathbb{Z}^{2}\right)$. The above operation, as opposed to a standard convolution, is not commutative; that is, $a \curvearrowleft b \neq b \curvearrowleft a$, since

$$
\begin{aligned}
(b \natural a)_{m, n} & =\sum_{k, l \in \mathbb{Z}} b_{k, l} a_{m-k, n-l} \omega^{(m-k) l} \\
& =\sum_{k, l \in \mathbb{Z}} a_{k, l} b_{m-k, n-l} \omega^{k(n-l)} .
\end{aligned}
$$

The transition from the invertibility of frame operators to the invertibility of twisted convolution follows from the observation that if $S$ and $T$ are two operators having Janssen representations of the form (6) corresponding to sequences $a$ and $b$, respectively, then

$$
\begin{aligned}
S T & =\left(\sum_{k, l \in \mathbb{Z}} a_{k, l} M_{k / \alpha} T_{l / \beta}\right)\left(\sum_{m, n \in \mathbb{Z}} b_{m, n} M_{m / \alpha} T_{n / \beta}\right) \\
& =\sum_{k, l \in \mathbb{Z}} \sum_{m, n \in \mathbb{Z}} a_{k, l} b_{m, n} M_{k / \alpha} T_{l / \beta} M_{m / \alpha} T_{n / \beta} \\
& =\sum_{k, l \in \mathbb{Z}} \sum_{m, n \in \mathbb{Z}} a_{k, l} b_{m, n} e^{-2 \pi i \frac{l}{\beta} \frac{m}{\alpha}} M_{(m+k) / \alpha} T_{(n+l) / \beta} \\
& =\sum_{k, l \in \mathbb{Z}} \sum_{m, n \in \mathbb{Z}} a_{k, l} b_{m, n} e^{-2 \pi i l m \frac{q}{p}} M_{(m+k) / \alpha} T_{(n+l) / \beta} \\
& =\sum_{m, n \in \mathbb{Z}} \sum_{k, l \in \mathbb{Z}} a_{k, l} b_{m-k, n-l} e^{\frac{-2 \pi i(m-k) l q}{p}} M_{m / \alpha} T_{n / \beta} \\
& =\sum_{m, n \in \mathbb{Z}}(a \curvearrowleft b)_{m, n} M_{m / \alpha} T_{n / \beta} .
\end{aligned}
$$

The sequence of the Janssen representation for the identity operator is the Dirac sequence $\delta$; hence, here it becomes evident how the twisted convolution comes into the game.
In [14], the authors give a necessary and sufficient condition on the Janssen coefficients of $S_{g, \gamma}$ to be invertible, based on a matrix valued function. The approach uses some sophisticated techniques of Gabor analysis mostly described in [7]. In the following, we present a more transparent (though equivalent) strategy that only deals with simple manipulations of the sequences. This method reveals further insights into Gabor frame theory and can be easily extended to the finite-dimensional setting leading to efficient concepts of algorithms for computing alternative dual Gabor elements.

\section{Twisted Convolution on $\mathbb{Z}_{\mathrm{p}} \times \mathbb{Z}_{\mathrm{p}}$}

The method that we discuss in the next section is heavily based on the properties of the twisted convolution on $\mathbb{Z}_{p} \times \mathbb{Z}_{p}$. In this simplified setting, we reveal the most important structures of the twisted convolution operation and emphasize those parts that are useful to understand our approach.

In this section, we describe twisted convolution on the finite group $F=\mathbb{Z}_{p} \times \mathbb{Z}_{p}$. The standard (commutative) convolution of two length $p^{2}$ sequences $f=\left(f_{k, l}\right)$ and $g=\left(g_{k, l}\right)$, with $k$, $l \in \mathbb{Z}_{p}$, is defined by

$$
(f * g)_{m, n}=\sum_{k, l=0}^{p-1} f_{k, l} g_{m-k, n-l}
$$

where operations on indices are performed modulo $p$.

By analogy with the infinite case, we define the twisted convolution $f\lfloor g$ of two elements $f$ and $g$ as above, by

$$
(f \curvearrowleft g)_{m, n}=\sum_{k, l=0}^{p-1} f_{k, l} g_{m-k, n-l} \omega^{(m-k) l}
$$

with $\omega=e^{-2 \pi i q / p}$. For a fixed $g$, the twisted convolution can be interpreted as a linear mapping $C_{g}: f \rightarrow f\lfloor g$ whose matrix $G$ is block circulant [see (2)] with $p$ blocks, i.e.,

$$
G=\mathcal{C}\left(G_{0}, G_{p-1}, \ldots, G_{1}\right)
$$

where each block is a $p \times p$ matrix with entries

$$
\left(G_{j}\right)_{k, l}=\omega^{j l} g_{j, k-l} \quad k, l=0, \ldots, p-1 .
$$

For example, when $p=3$, the blocks have the form

$$
G_{j}=\left(\begin{array}{ccc}
g_{j, 0} & \omega^{j} g_{j, 2} & \omega^{2 j} g_{j, 1} \\
g_{j, 1} & \omega^{j} g_{j, 0} & \omega^{2 j} g_{j, 2} \\
g_{j, 2} & \omega^{j} g_{j, 1} & \omega^{2 j} g_{j, 0}
\end{array}\right), \quad j=0,1,2 .
$$

In the case of the standard convolution, that is, when $p=q$, the blocks $G_{j}$ of the matrix $G$ are circulant themselves, with entries

$$
\left(G_{j}\right)_{k, l}=g_{j, k-l}, \quad k, l=0, \ldots, p-1 .
$$

When $p=3$, we obtain

$$
G_{j}=\left(\begin{array}{ccc}
g_{j, 0} & g_{j, 2} & g_{j, 1} \\
g_{j, 1} & g_{j, 0} & g_{j, 2} \\
g_{j, 2} & g_{j, 1} & g_{j, 0}
\end{array}\right), \quad j=0,1,2 .
$$


For inverting block circulant matrices, we use the Fourier transform.

Lemma 4.1: The matrix $G=\mathcal{C}\left(G_{0}, G_{p-1}, \ldots, G_{1}\right)$ is invertible if and only if for every $s=0, \ldots, p-1$, $\widehat{G}_{s}=\sum_{r=0}^{p-1} e^{-2 \pi i s r / p} G_{r}$ is invertible. In this case, the inverse of $G$ is

$$
G^{-1}=\mathcal{C}\left(H_{0}, H_{p-1}, \ldots, H_{1}\right)
$$

with

$$
H_{r}=\frac{1}{p} \sum_{s=0}^{p-1} e^{2 \pi i s r / p}\left(\widehat{G}_{s}\right)^{-1}
$$

In principle, we need to study invertibility of each $\widehat{G}_{s}$, for $s=0, \ldots, p-1$. However, due to the structure of the blocks in a twisted convolution matrix, it suffices to look only at $\widehat{G}_{0}$.

Lemma 4.2: Let $G=\mathcal{C}\left(G_{0}, G_{p-1}, \ldots, G_{1}\right)$ be a twisted convolution matrix defined by (9). For $s 1, \ldots, p-1, \widehat{G}_{s}$ (as defined in Lemma 4.1) is invertible if and only if $\widehat{G}_{0}$ is invertible.

Proof: For $r, s=0, \ldots, p-1$

$$
\begin{aligned}
\left(\widehat{G}_{s+r q}\right)_{n, l} & =\sum_{k=0}^{p-1} e^{-2 \pi i(s+r q) k / p}\left(G_{k}\right)_{n, l} \\
& =\sum_{k=0}^{p-1} e^{-2 \pi i(s+r q) k / p} \omega^{k l} g_{k, n-l} \\
& =\sum_{k=0}^{p-1} e^{-2 \pi i s k / p} \omega^{k(l+r)} g_{k,(n+r)-(l+r)} \\
& =\sum_{k=0}^{p-1} e^{-2 \pi i s k / p}\left(G_{k}\right)_{n+r, l+r} \\
& =\left(\widehat{G}_{s}\right)_{n+r, l+r} .
\end{aligned}
$$

That is, all blocks are unitarily equivalent, in the sense that

$$
T_{r} \widehat{G}_{s} T_{r}^{*}=\widehat{G}_{s+q r}
$$

where $T_{r}$ denotes the unitary matrix with entries

$$
\left(T_{r}\right)_{k, l}= \begin{cases}1, & \text { if } p-r=l-k \\ 0, & \text { otherwise. }\end{cases}
$$

Since $p$ and $q$ are relatively prime, we obtain all blocks by such unitary transformations. This implies that if $\widehat{G}_{0}$ is invertible, then all $\widehat{G}_{s}$ are invertible for $s=1, \ldots, p-1$.

In other words, the $p \times p$ matrix $\widehat{G}_{0}$ contains all the information about the invertibility of $C_{g}$ [defined under (8)]. From (10), the entries of $\widehat{G}_{0}$ are given by

$$
\left(\widehat{G}_{0}\right)_{n, l}=\sum_{k=0}^{p-1} \omega^{k l} g_{k, n-l} .
$$

As an example, for $p=3$, we have

$$
\widehat{G}_{0}=\left(\begin{array}{ccc}
\sum_{k=0}^{2} g_{k, 0} & \sum_{k=0}^{2} \omega^{k} g_{k, 2} & \sum_{k=0}^{2} \omega^{2 k} g_{k, 1} \\
\sum_{k=0}^{2} g_{k, 1} & \sum_{k=0}^{2} \omega^{k} g_{k, 0} & \sum_{k=0}^{2} \omega^{2 k} g_{k, 0} \\
\sum_{k=0}^{2} g_{k, 2} & \sum_{k=0}^{2} \omega^{k} g_{k, 1} & \sum_{k=0}^{2} \omega^{2 k} g_{k, 0}
\end{array}\right) .
$$

We will see in Section $V$ that this observation leads to the matrix algebra that we introduce to study the invertibility of the twisted convolution for both the continuous and the discrete setting.

\section{Main Theoretical Results}

As seen in Section III, we can transfer the invertibility problem of a Gabor frame-type operator to the invertibility of its Janssen coefficient sequence with respect to the twisted convolution. In this section, we describe a constructive approach that shows how one can verify the invertibility of such a sequence and build its inverse.

Let $a=\left(a_{m, n}\right)$ be a sequence in $\ell^{1}\left(\mathbb{Z}^{2}\right)$ and $S$ an operator with Janssen coefficient sequence $a$, that is, $S \sum_{m, n \in \mathbb{Z}} a_{m, n} M_{m / \alpha} T_{n / \beta}$. Our aim is to describe a method for verifying if $S$ is invertible. However, as we saw in Section III, this boils down to checking if there exists an inverse sequence $b$ of $a$ in $\left(\ell^{1}\left(\mathbb{Z}^{2}\right), \nvdash\right)$. However, since $\measuredangle$ is not a commutative operation we cannot use standard Fourier transform techniques. Instead, we define a map $\phi$ that takes a sequence $a \in \ell^{1}\left(\mathbb{Z}^{2}\right)$ to a special matrix algebra consisting of $p \times p$ matrices whose entries are sequences in $\ell^{1}\left(\mathbb{Z}^{2}\right)$. We denote this algebra by $(\mathcal{M}, *)$. Thanks to this mapping the problem of inverting $a$ in $\left(\ell^{1}, \measuredangle\right)$ reduces to inverting a $p \times p$ matrix $\phi(a)$ in $(\mathcal{M}, *)$. Now we outline the main components of the theory in order to be able to apply the constructive methods to concrete examples.

The goal is to reduce twisted convolution on $\mathbb{Z} \times \mathbb{Z}$ to twisted convolution on $\mathbb{Z}_{p} \times \mathbb{Z}_{p}$. To do so, for $a \in \ell^{1}\left(\mathbb{Z}^{2}\right)$, we define

$$
\left(a^{r, s}\right)_{k, l}= \begin{cases}a_{k, l}, & \text { if }(k, l) \equiv_{p}(r, s) \\ 0, & \text { else }\end{cases}
$$

where $r, s \in \mathbb{Z}_{p}$ and the symbol $\equiv_{p}$ denotes equivalence modulo $p$. We see that $a^{r, s}$ is supported on the coset $(r+p \mathbb{Z}) \times(s+$ $p \mathbb{Z})$ and $a=\sum_{r, s \in \mathbb{Z}_{p}} a^{r, s}$. In the case when $p=2$, out of a sequence $a$ we obtain four subsequences $a^{0,0}$ which is supported on $2 \mathbb{Z} \times 2 \mathbb{Z}, a^{0,1}$ which is supported on $2 \mathbb{Z} \times(2 \mathbb{Z}+1), a^{1,0}$ supported on $(2 \mathbb{Z}+1) \times 2 \mathbb{Z}$ and $a^{1,1}$ supported on $(2 \mathbb{Z}+1) \times$ $(2 \mathbb{Z}+1)$. With definition (13), we obtain for $u, v \in \mathbb{Z}_{p}$

$$
\begin{aligned}
(a & \downarrow b)_{u+p \mathbb{Z}, v+p \mathbb{Z}} \\
& =\sum_{k, l \in \mathbb{Z}} a_{k, l} b_{u+p \mathbb{Z}-k, v+p \mathbb{Z}-l} \omega^{(u-k) l} \\
& =\sum_{r, s \in \mathbb{Z}_{p}} \sum_{(k, l) \equiv_{p}(r, s)} a_{k, l} b_{u+p \mathbb{Z}-k, v+p \mathbb{Z}-l} \omega^{(u-r) s} \\
& =\sum_{r, s \in \mathbb{Z}_{p}} \sum_{k, l \in \mathbb{Z}}\left(a^{r, s}\right)_{k, l}\left(b^{u-r, v-s}\right)_{u+p \mathbb{Z}-k, v+p \mathbb{Z}-l} \omega^{(u-r) s} \\
& =\sum_{r, s \in \mathbb{Z}_{p}}\left(a^{r, s} * b^{u-r, v-s}\right)_{u+p \mathbb{Z}, v+p \mathbb{Z} \omega^{(u-r) s} .}
\end{aligned}
$$

In a more compact notation, we have

$$
(a \bigsqcup b)^{u, v}=\sum_{r, s=0}^{p-1} a^{r, s} * b^{u-r, v-s} \omega^{(u-r) s}, u, v \in \mathbb{Z}_{p} .
$$

We observe that (14) resembles (8) with the only difference that the components in the sum are sequences in (14) and numbers in (8). Hence, the twisted convolution can be transformed into 
a twisted convolution over $\mathbb{Z}_{p} \times \mathbb{Z}_{p}$ with sequences as elements and standard convolution instead of multiplication. We saw in Section III that invertibility of an element with respect to twisted convolution is equivalent to checking invertibility of the matrix (12). In the following, motivated by the circulant structure of the twisted convolution matrix in $\mathbb{Z}_{p} \times \mathbb{Z}_{p}$, we introduce a new matrix algebra whose entries are sequences which is isomorphic to $\left(\ell^{1}\left(\mathbb{Z}^{2}\right), \downarrow\right)$.

Let $(\mathcal{M}, *)$ be an algebra of $(p \times p)$-matrices whose entries are $\ell^{1}$-sequences and multiplication of two elements $A, B \in \mathcal{M}$ is given by

$$
(A * B)_{r, s}=\sum_{l \in \mathbb{Z}_{p}} A_{r, l} * B_{l, s}, \quad r, s \in \mathbb{Z}_{p} .
$$

Note that $A_{r, l}$ and $B_{l, s}$ are sequences. The identity element Id is a matrix with $\delta$ sequences on the diagonal. In analogy to (12), we define a mapping $\phi:\left(\ell^{1}, \downarrow\right) \rightarrow(\mathcal{M}, *)$ by

$$
(\phi(a))_{r, s}=\sum_{m \in \mathbb{Z}_{p}} \omega^{m s} a^{m, r-s}
$$

where we map a sequence to a matrix whose elements are sequences. For example, when $\alpha \beta=2 / q$, that is $p=2$, and $q \in \mathbb{N}$ relatively prime to $p$, the above matrix takes the form

$$
\begin{aligned}
\phi(a) & =\left(\begin{array}{ll}
a^{0,0}+a^{1,0} & a^{0,1}+\omega a^{1,1} \\
a^{0,1}+a^{1,1} & a^{0,0}+\omega a^{1,0}
\end{array}\right) \\
& =\left(\begin{array}{ll}
a^{0,0}+a^{1,0} & a^{0,1}-a^{1,1} \\
a^{0,1}+a^{1,1} & a^{0,0}-a^{1,0}
\end{array}\right)
\end{aligned}
$$

where we used the fact that $\omega=e^{-2 \pi i q / p}=-1$. Note that summing up the elements of the first column gives us back the sequence $a$.

The mapping $\phi$ is linear, and $(\phi(\delta))_{r, s}=\sum_{m=0}^{p-1} \omega^{m s} \delta^{m, r-s}$ implying that $\phi(\delta) \mathrm{Id}$. We observe that the matrix $\phi(a)$ defined in (15) resembles in structure the matrix $\widehat{G}_{0}$ defined in (12). The important property of $\phi$, shown in [5], is that $\phi$ is an antihomomorphism, i.e.,

$$
\phi(a \bigsqcup b)=\phi(b) * \phi(a) .
$$

For the problem of invertibility of a sequence $a \in \ell^{1}$, this means that if $a$ is invertible in $\left(\ell^{1}\left(\mathbb{Z}^{2}\right), \downarrow\right)$, then $\phi(a)$ is invertible in $(\mathcal{M}, *)$. Indeed, suppose that $a$ is invertible in $\left(\ell^{1}, \downarrow\right)$. Then

$$
\mathrm{Id}=\phi(\delta)=\phi\left(a^{-1} \sqsubset a\right) \phi(a) * \phi\left(a^{-1}\right)
$$

which means that $\phi(a)$ is invertible in $(\mathcal{M}, *)$, with inverse equal to $\phi\left(a^{-1}\right)$. What we need for our problem is the reverse implication, that is to show that if $A=\phi(a)$ is invertible, then so is $a$. Since the matrix $A$ has a special form, namely the entries are coming from a sequence $a$, we need to make sure that the entries of $A^{-1}$ are also coming from some sequence $b \in \ell^{1}$, that is that $A^{-1}=\phi(b)$. Then, since Id $=\phi(a) * \phi(b)=\phi(b \llbracket a), b$ will be the desired inverse of $a$, because $b \downarrow a=\delta$. The problem of the inverse matrix to have the same structure as the original one, is usually referred to as Wiener's Lemma. In the simplest way, it says that if some mathematical object $A$ of special type is invertible, then its inverse $A^{-1}$ is also of the same special type. With this observation and the following theorem, we have all components in order to verify the invertibility of a twisted convolution operator.

Theorem 5.1 [Wiener's Lemma] [5] : Let $A=\phi(a)$ for some $a \in \ell^{1}$. If $A$ is invertible in $\mathcal{M}$, then there exists a unique $b \in \ell^{1}$ such that $\phi(b)=A^{-1} \in \mathcal{M}$.

The proof is constructive and the sketch can be found in Appendix A.

Theorem 5.1 provides the key result to study invertibility of twisted convolution. Indeed, for a given sequence $a$ in $\ell^{1}$, we look at the corresponding matrix $A=\phi(a)$ as defined in (15). First, to check if $A$ is invertible, we compute the determinant of $A$. We do it just like in the case when the entries of the matrix are numbers. Here, we have to keep in mind that the entries are sequences and the "multiplication" of two sequences is just convolution. For example, when $p=2, A$ is given by (16), and

$\operatorname{det} A=\left(a^{0,0}+a^{1,0}\right) *\left(a^{0,0}-a^{1,0}\right)-\left(a^{0,1}+a^{1,1}\right) *\left(a^{0,1}-a^{1,1}\right)$.

If $A$ is invertible in $(\mathcal{M}, *)$; that is, $\operatorname{det} A$ is an invertible sequence in $\left(\ell^{1}\left(\mathbb{Z}^{2}\right), *\right)$, then the inverse of $A$ can be computed using Cramer's rule

$$
\left(A^{-1}\right)_{r, s}=(\operatorname{det} A)^{-1} * \operatorname{det} A(s, r)
$$

where $A(s, r)$ is a $p \times p$ matrix obtained from $A$ by substituting the $s$ th row of $A$ with a vector of zeros having $\delta$ on the $r$ th position, and the $r$ th column with a column of zeros having $\delta$ on the $s$ th position. When $p=2$, we get

$$
\begin{aligned}
& A 1=\left(\begin{array}{ll}
\delta & 0 \\
0 & a^{0,0}-a^{1,0}
\end{array}\right) A 2=\left(\begin{array}{cc}
0 & \delta \\
a^{0,1}+a^{1,1} & 0
\end{array}\right) \\
& A 3=\left(\begin{array}{ll}
0 & a^{0,1}-a^{1,1} \\
\delta & 0
\end{array}\right) A 4=\left(\begin{array}{cc}
a^{0,0}+a^{1,0} & 0 \\
0 & \delta
\end{array}\right)
\end{aligned}
$$

where $A 1=A(0,0), A 2=A(1,0), A 3=A(0,1)$, and $A 4=$ $A(1,1)$. Hence

$$
A^{-1}=(\operatorname{det} A)^{-1} *\left(\begin{array}{cc}
a^{0,0}-a^{1,0} & -a^{0,1}+a^{1,1} \\
-a^{0,1}-a^{1,1} & a^{0,0}+a^{1,0}
\end{array}\right) .
$$

The approach is constructive in the sense that algebraic methods such as Cramer's Rule can be applied to find the inverse of $A$. To matrix $A^{-1}$, according to Theorem 5.1, corresponds another element $b$ in $\ell^{1}$, that is $A^{-1} \phi(b)$. This element, in turn, provides the inverse of $a$ in $\left(\ell^{1}, t\right)$. The sequence $b$ can simply be read from the entries of $A^{-1}$ according to the mapping $\phi$, that is we obtain $b$ by summing up the elements from the first column of $A^{-1}$. Therefore, it is enough to compute only the first column of the inverse matrix $A^{-1}$. In our example, $p=2$, the sequence $b$ is equal to $(\operatorname{det} A)^{-1} *\left(a^{0,0}-a^{1,0}-a^{0,1}-a^{1,1}\right)$. For small $p$, this method leads to fast inversion schemes for the twisted convolution operator. In the last section, we will show how this approach is used in the setting of finite 1-D signals.

In the case of integer oversampling, that is, when $(\alpha \beta)^{-1} \in$ $\mathbb{N}$, implying $p=1$, the matrix $A=\phi(a)$ reduces to a sequence $a$ itself, since $r=s=0$. Then the invertibility of the operator $S$ is equivalent to the invertibility of the sequence $a$ in $\left(\ell^{1}\left(\mathbb{Z}^{2}\right), *\right)$.

\section{EQuivalence TO OTHER KNOWN METHODS}

In this section, we describe two known methods dealing with the characterization of invertibility of frame-type opera- 
tors $S_{g, \gamma}$, where $g, \gamma \in L^{2}(\mathbb{R})$, and their equivalence to our approach. We consider only those functions $g$ and $\gamma$ such that $\left\langle\gamma, M_{m / \alpha} T_{n / \beta} g\right\rangle, m, n \in \mathbb{Z}$, is an $\ell^{1}$ sequence. A class of functions with this property is the Feichtinger algebra $S_{0}$ defined in Section V. Furthermore, since we assume that $\mathcal{G}(g, \alpha, \beta)$ and $\mathcal{G}(\gamma, \alpha, \beta)$ form Gabor frames, $\alpha \beta=p / q \leq 1$.

The first approach uses sophisticated methods from Gabor analysis, and the second makes use of the vector-valued Zak transform and its properties [7].

\section{A. Approach Using Gabor Analysis Tools}

This approach was developed by the authors in [14], and is based on time-frequency methods. We assume that $\alpha \beta p / q$ and that $g, \gamma \in S_{0}$. Define the bi-infinite matrix valued function $G(x)$ by the correlation functions

$$
G_{j, l}(x)=\sum_{k \in \mathbb{Z}} \overline{g(x-l / \beta-\alpha k)} \gamma(x-j / \beta-\alpha k), \quad j, l \in \mathbb{Z} .
$$

Because $g, \gamma \in S_{0}, T_{l / \beta} \bar{g} \cdot T_{j / \beta} \gamma$ is in $S_{0}$, since $S_{0}$ is closed under translation and under pointwise multiplication [7]. Moreover, the periodization $G_{j, l}(x)$ of period $\alpha$ is continuous. As shown in [7, Ch. 6] by virtue of Schur's test, the bi-infinite matrix $G(x)$ is a bounded operator on $\ell^{2}(\mathbb{Z})$ for all $x$. We can also define $G(x)$ by the Fourier series

$$
G_{0, n}(x)=\alpha^{-1} \sum_{m \in \mathbb{Z}} a_{m, n} e^{2 \pi i m x / \alpha}
$$

where $a_{m, n}=\left\langle\gamma, M_{m / \alpha} T_{n / \beta} g\right\rangle$, and use the fact that $G_{j, l}(x)=G_{0, l-j}(x-j / \beta)$ to extend it to $G_{j, n}(x)$.

The correlation functions $G_{0, n}(x)$ provide an important representation of the frame-type operator $S_{g, \gamma}$, the so-called Walnut representation

$$
S_{g, \gamma} f=\alpha \sum_{n \in \mathbb{Z}} G_{0, n} T_{n / \beta} f, \quad f \in L^{2}
$$

that follows directly from the Janssen representation (6) of $S_{g, \gamma}$. Since $g, \gamma \in S_{0}$, the series of the Walnut representation converges unconditionally in $L^{2}$ [7]. Next, we define the entries of the $(p \times p)$-matrix valued function $\Psi(\xi, x)\left(\psi_{r, s},(\xi, x)\right)_{r, s=0}^{p-1}$ by

$$
\psi_{r, s}(\xi, x)=\alpha^{-1} \sum_{m, n \in \mathbb{Z}} a_{m, s-r-p n} e^{\frac{-2 \pi i m r}{\alpha \beta}} e^{2 \pi i\left(\frac{m x}{\alpha}+\frac{n \xi}{\beta}\right)} .
$$

As shown in [14], the frame operator $S_{g, \gamma}$ is invertible if and only if $\operatorname{det}(\Psi(\xi, x)) \neq 0$ for all $(\xi, x)$.

In the case of integer oversampling, the matrix $\Psi$ simply becomes

$$
\psi(\xi, x)=\alpha^{-1} \sum_{m, n \in \mathbb{Z}} a_{m, n} e^{2 \pi i(m x / \alpha+n \xi / \beta)} .
$$

That is, $\psi$ is the inverse Fourier transform of the sequence $a$. This implies that $S$ is invertible if and only if the sequence $a$ is invertible in $\left(\ell^{1}\left(\mathbb{Z}^{2}\right), *\right)$, and this approach coincides with our method. Theorem 6.1 below shows that not only in integer oversampling, but also in the general rational case, the matrix $\Psi(\xi, x)$ is equivalent to the matrix $A$, described in the previous section, via the Fourier transform.
Theorem 6.1: Let $g, \gamma \in S_{0}, \alpha \beta=p / q$ and $a_{m, n}\left\langle\gamma, M_{m / \alpha} T_{n / \beta} g\right\rangle$. Then

$$
\mathcal{F} \psi_{r, s}=\beta A_{s, r} \quad \text { for } \quad r, s=0, \ldots, p-1
$$

where $A$ is a matrix with entries given by $A_{r, s} \sum_{k=0}^{p-1} \omega^{k s} a^{k, r-s}$. Moreover, $\mathcal{F} \operatorname{det} \Psi=\beta^{p} \operatorname{det} A$.

For the proof, we refer the reader to Appendix B.

Theorem 6.1 provides an equivalence between two invertibility characterizations in the sense that $\operatorname{det}(\Psi(\xi, x)) \neq 0$ if and only if $\operatorname{det} A$ is invertible in $\left(\ell^{1}, *\right)$. However, whereas our approach applies also to the finite-dimensional discrete setting, this is not the case in [14]. There the authors considered this problem not from the point of view of Janssen representation but taking advantage of the structure of the Gabor matrix.

\section{B. Zibulski-Zeevi Representation}

Another characterization of invertibility of the frame operator $S_{g, \gamma}$ is due to Zibulski and Zeevi and relies on the notion of the Zak transform. Given $g, \gamma \in L^{2}(\mathbb{R})$, the Zak transform of $g$ is defined as

$$
\mathcal{Z}_{\alpha} g(x, \xi)=\sum_{k \in \mathbb{Z}} g(x-\alpha k) e^{2 \pi i \alpha k \xi}
$$

The Zak transform is a unitary mapping from $L^{2}(\mathbb{R})$ into $L^{2}\left([0, \alpha]^{2}\right)$, that is

$$
\alpha\left\langle\mathcal{Z}_{\alpha} \gamma, \mathcal{Z}_{\alpha} g\right\rangle_{L^{2}\left([0, \alpha]^{2}\right)}=\langle\gamma, g\rangle_{L^{2}(\mathbb{R})}
$$

and satisfies the following relation with respect to time-frequency shifts, for $k, l \in \mathbb{Z}$

$$
\mathcal{Z}_{\alpha}\left(M_{k / \alpha} T_{l / \beta}\right)(x, \xi)=e^{2 \pi i x k / \alpha} e^{-2 \pi i \xi l / \beta} \mathcal{Z}_{\alpha} g(x, \xi) .
$$

For $j \in\{0, \ldots, p-1\}, x \in[0, \alpha / p]$ and $\xi \in[0,1 / \alpha]$, we consider the vector-valued Zak transform

$$
\left(\mathcal{Z}_{\alpha} g(x, \xi)\right)_{j}=\mathcal{Z}_{\alpha} g(x+\alpha j / p, \xi)
$$

and introduce the matrix-valued function $\mathcal{A}(x, \xi)$ over the index set $\{0, \ldots, p-1\}$ defined by the entries

$$
\begin{aligned}
& \mathcal{A}_{r, s}(x, \xi)=\alpha \sum_{j=0}^{q-1} \frac{\mathcal{Z}_{\alpha} g(x+\alpha s / p, \xi-\beta j)}{} \\
& \quad \times \mathcal{Z}_{\alpha} \gamma(x+\alpha r / p, \xi-\beta j) e^{2 \pi i j(r-s) / q}
\end{aligned}
$$

Then Zibulski-Zeevi representation states that

$$
S_{g, \gamma}=\mathcal{Z}_{\alpha}^{-1} \mathcal{A} \mathcal{Z}_{\alpha}
$$

and, hence, $S_{g, \gamma}$ is invertible if and only if the matrix-valued function $\mathcal{A}(x, \xi)$ is invertible for almost all $(x, \xi) \in[0, \alpha / p] \times$ $[0,1 / \alpha]$.

In the case of the integer oversampling, $p=1$ and $\alpha q=$ $1 / \beta$, the matrix-valued function $\mathcal{A}$ becomes a complex valued function

$$
\mathcal{A}(x, \xi)=\alpha \sum_{j=0}^{q-1} \overline{\mathcal{Z}_{\alpha} g(x, \xi-\beta j)} \mathcal{Z}_{\alpha} \gamma(x, \xi-\beta j)
$$


on $[0, \alpha] \times[0,1 / \alpha q]$. By taking the Fourier transform of $\mathcal{A}$ and using (21) and (22), we obtain

$$
\begin{aligned}
\mathcal{F} \mathcal{A}(k, l)= & \alpha \int_{0}^{\alpha} \int_{0}^{\frac{q}{\alpha}} \sum_{j=0}^{q-1} \overline{\mathcal{Z}_{\alpha} g(x, \xi-\beta j)} \mathcal{Z}_{\alpha} \gamma(x, \xi-\beta j) \\
& \times e^{\frac{-2 \pi i x k}{\alpha}} e^{\frac{-2 \pi i \xi l}{\beta}} d x d \xi \\
= & \alpha \int_{0}^{\alpha} \int_{0}^{1 / \alpha} \overline{\mathcal{Z}_{\alpha} g(x, \xi)} \mathcal{Z}_{\alpha} \gamma(x, \xi) e^{\frac{-2 \pi i x k}{\alpha}} e^{\frac{-2 \pi i \xi l}{\beta}} d x d \xi \\
= & \alpha \int_{0}^{\alpha} \int_{0}^{1 / \alpha} \overline{\mathcal{Z}_{\alpha}\left(M_{k / \alpha} T_{-l / \beta} g\right)(x, \xi)} \mathcal{Z}_{\alpha} \gamma(x, \xi) d x d \xi \\
= & \alpha\left\langle\mathcal{Z}_{\alpha} \gamma, \mathcal{Z}_{\alpha}\left(M_{k / \alpha} T_{-l / \beta} g\right)\right\rangle_{L^{2}\left([0, \alpha]^{2}\right)} \\
= & \left\langle\gamma, M_{k / \alpha} T_{-l / \beta} g\right\rangle_{L^{2}(\mathbb{R})}=a_{k,-l .} .
\end{aligned}
$$

Thus, the inversion of the frame operator reduces to inverting $a$ in $\left(\ell^{1}, *\right)$.

In the more general setting, that is, for any rational oversampling, the Zibulski-Zeevi matrix is equivalent to the matrix $\Psi$ described in the previous section.

Theorem 6.2: Let $g, \gamma \in L^{2}(\mathbb{R})$ and $\alpha \beta=p / q$. Then for all $(x, \xi) \in[0, \alpha / p] \times[0,1 / \alpha]$ and $r, s=0, \ldots, p-1$

$$
\mathcal{A}_{r, s}(x, \xi)=\alpha q e^{2 \pi i k_{0}(r-s) \alpha \xi} \psi_{-r l_{0},-s l_{0}}(p \xi, x)
$$

where $\psi_{r, s}(\xi, x)$ is defined in (20), and $k_{0}, l_{0} \in \mathbb{Z}$ are such that $p k_{0}+q l_{0}=1$. Moreover, $\operatorname{det} \mathcal{A}(x, \xi) \neq 0$ if and only if $\operatorname{det} \Psi(p \xi, x) \neq 0$ for almost all $(x, \xi)$.

The proof is presented in Appendix C.

Theorem 6.2 implies that the Zibulski-Zeevi condition on invertibility of $S_{g, \gamma}$ is equivalent to the one described in Section IV, by Theorem 6.1.

The benefit of our approach is that the inverse of a matrix $A$ gives us back in a constructive manner an inverse of the sequence $a$ in $\left(\ell^{1}, \downarrow\right)$. Second, this new approach works for the continuous as well as the discrete case, with no need to distinction as it was in [14]. The finite discrete setting is worked out in the next section.

\section{GABOR FRAME-TYPE OPERATOR FOR FINITE-LENGTH SIGNALS}

In this section, we will illustrate in more detail how the method described in Section V, more precisely the division of the sequence $a$ into smaller sequences, looks like when we consider Gabor analysis on a space of finite-dimensional signals.

Thus, we now consider Gabor analysis of sequences of length $L$. The goal is to determine if the frame-type operator $S_{g, \gamma}$, which we denote by $S$, is invertible and if so then to construct the inverse. We can describe $S$ by its Janssen representation associated to vectors $g, \gamma \in \mathbb{C}^{L}$ and parameters $\alpha$ and $\beta$, where we assume that $\alpha$ and $\beta$ divide $L$ and $\alpha \beta / L=p / q \leq 1$, with $p$ and $q$ relatively prime. The Janssen representation of the operator $S$ (as described in Section V) is

$$
S=\sum_{(m, n) \in \mathbb{Z}_{\beta} \times \mathbb{Z}_{\alpha}} a_{m, n} M_{m L / \alpha} T_{n L / \beta}
$$

where $a_{m, n}=\left\langle\gamma M_{m L / \beta} T_{n L / \alpha} g\right\rangle$ for all $(m, n) \in \mathbb{Z}_{\beta} \times \mathbb{Z}_{\alpha}$. We define the set of sequences with index set $\mathbb{Z}_{\beta} \times \mathbb{Z}_{\alpha}$ by $\ell^{1}\left(\mathbb{Z}_{\beta} \times\right.$ $\mathbb{Z}_{\alpha}$ ). As discussed in Section V, if $S$ is invertible, then there is a sequence $b \in \ell^{1}\left(\mathbb{Z}_{\beta} \times \mathbb{Z}_{\alpha}\right)$ such that $a\lfloor b=\delta$, and the inverse of $S$ is the operator $T=\sum_{(m, n) \in \mathbb{Z}_{\beta} \times \mathbb{Z}_{\alpha}} b_{m, n} M_{m L / \alpha} T_{n L / \beta}$. First, we present the method for general $p$ and then show an explicit example when $p=2$.

Let $a, b \in \ell^{1}\left(\mathbb{Z}_{\beta} \times \mathbb{Z}_{\alpha}\right)$. Then the standard convolution between $a$ and $b$ is defined as

$$
(a * b)_{m, n}=\sum_{k, l \in \mathbb{Z}_{\beta} \times \mathbb{Z}_{\alpha}} a_{k, l} b_{m-k, n-l} \quad m, n \in \mathbb{Z}_{\beta} \times \mathbb{Z}_{\alpha}
$$

where operation on indices is performed modulo $(\beta, \alpha)$. By analogy with the infinite case, we define the twisted convolution $a \curvearrowleft b$ of two elements $a, b \in \ell^{1}\left(\mathbb{Z}_{\beta} \times \mathbb{Z}_{\alpha}\right)$ by

$$
(a \curvearrowleft b)_{m, n}=\sum_{k, l \in \mathbb{Z}_{\beta} \times \mathbb{Z}_{\alpha}} a_{k, l} b_{m-k, n-l} \omega^{(m-k) l}
$$

with $m, n \in \mathbb{Z}_{\beta} \times \mathbb{Z}_{\alpha}$, where again the operation on indices is performed modulo $(\beta, \alpha)$, and $\omega=e^{-2 \pi i p / q}$, with $p, q$ relatively prime and $p$ dividing $L, \alpha$ and $\beta$.

Let $a$ be a sequence of Janssen coefficients representing $S$. We want to see if $S$ is invertible, and if so, then to construct the inverse. Invertibility of $S$ is equivalent to the invertibility of the sequence $a$ with respect to twisted convolution. Hence, we use the techniques described in Section V.

Since $p$ divides $\alpha$ and $\beta, \mathbb{Z}_{\beta} \times \mathbb{Z}_{\alpha}$ admits a decomposition into $p^{2}$ disjoint subsets $(r, s)+\left(p \mathbb{Z}_{\beta} \times p \mathbb{Z}_{\alpha}\right)$, where $r, s \in \mathbb{Z} p$. Namely

$$
\mathbb{Z}_{\beta} \times \mathbb{Z}_{\alpha}=\bigcup_{r, s=0}^{p-1}(r, s)+\left(p \mathbb{Z}_{\beta} \times p \mathbb{Z}_{\alpha}\right) .
$$

Therefore, we can define subsequences of $a$ in a similar manner as in (13)

$$
\left(a^{r, s}\right)_{m, n}= \begin{cases}a_{m, n}, & \text { if }(m, n) \equiv_{p}(r, s) \\ 0, & \text { else. }\end{cases}
$$

The construction for $p=2$ is pictured in Fig. 2 for a random sequence $a \in \ell^{1}\left(\mathbb{Z}_{8} \times \mathbb{Z}_{8}\right)$ (Fig. 1).

We now consider an explicit example. We will use the method from Section V to check if the given Gabor frame-like operator is invertible. This example is analogous to the one considered in Section $\mathrm{V}$, but the sequences there were infinite length.

Let $L=2^{9} \cdot 3, \alpha=\beta=2^{5}$ and let $g, \gamma$ be two Gaussians of different spread, that is

$$
g[k]=e^{-k^{2}}, \quad \text { and } \quad \gamma[k]=e^{-k^{2} / 2} .
$$

Since $\alpha \beta / L=2 / 3, p=2$ and the matrix $A=$ $\phi(a)$ corresponding to a Janssen representation sequence $a_{m, n}\left\langle\gamma, M_{3 \cdot 2^{4} m} T_{3 \cdot 2^{4} n} g\right\rangle$ of a Gabor frame-like operator $S_{g, \gamma}$ is a $2 \times 2$ matrix of the form (16), with the difference that now the entries of the matrix are finite length $\alpha \beta=2^{10}$ sequences. The 2-D Fourier transform of $\operatorname{det} A$ (Fig. 3 ) has no zeros; therefore, $\operatorname{det} A$ is an invertible sequence in $\left(\ell^{1}\left(\mathbb{Z}_{\beta} \times \mathbb{Z}_{\alpha}\right), *\right)$. This implies, by Wiener's Lemma (Theorem 5.1), that $a$ has 


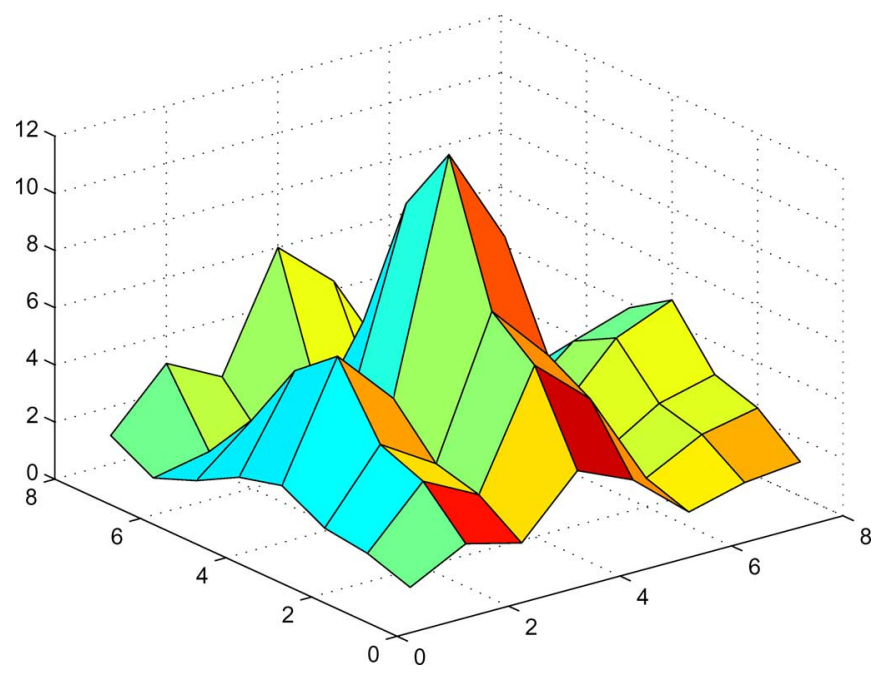

Fig. 1. Random 2-D sequence $a$ of size $8 \times 8$. a)

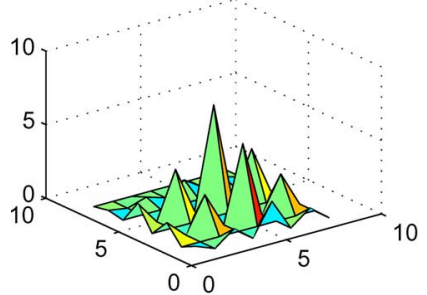

c)

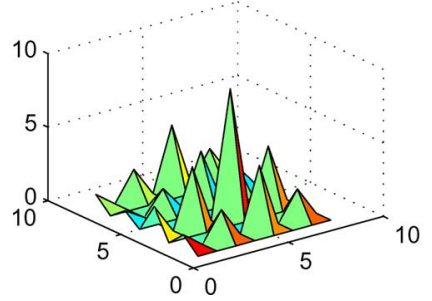

b)

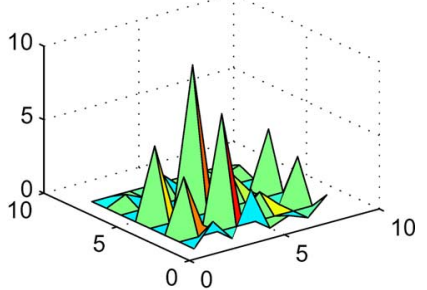

d)

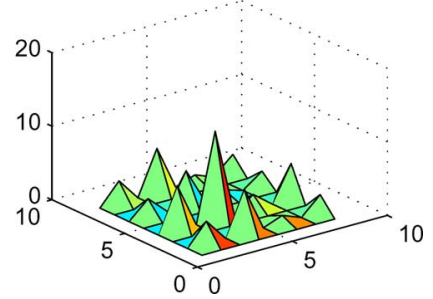

Fig. 2. Decomposition of signal $a$ into four $(p=2)$ subsequences: (a) $a^{00}$, (b) $a^{10}$, (c) $a^{01}$, and (d) $a^{11}$.

an inverse with respect to the twisted convolution, namely a sequence

$$
b=(\operatorname{det} A)^{-1} *\left(a^{0,0}-a^{1,0}-a^{0,1}-a^{1,1}\right) .
$$

This shows that inverting a sequence $a$ in $\left(\ell^{1}\left(\mathbb{Z}_{\beta} \times \mathbb{Z}_{\alpha}\right),\llcorner)\right.$ essentially boils down to inverting a sequence, namely $\operatorname{det} A$, in $\left(\ell^{1}\left(\mathbb{Z}_{\beta} \times \mathbb{Z}_{\alpha}\right), *\right)$, for which fast Fourier transform schemes can be applied.

Here, we presented a method of inverting Gabor frame operators given by the Janssen representation. There exist many other methods of inverting Gabor frame operators in the case of finite-length signals that take advantage of a highly structured matrix form that the operator $S$ admits [13], [14]. However, the previous methods apply only when considering finite discrete models and there is no analogue of them in the continuous setting. The method that we presented here covers both environments (since it is not based on $S$ directly, but rather on the Janssen coefficient sequence associated to $S$ ), thus giving a smooth transition between the continuous and discrete settings.

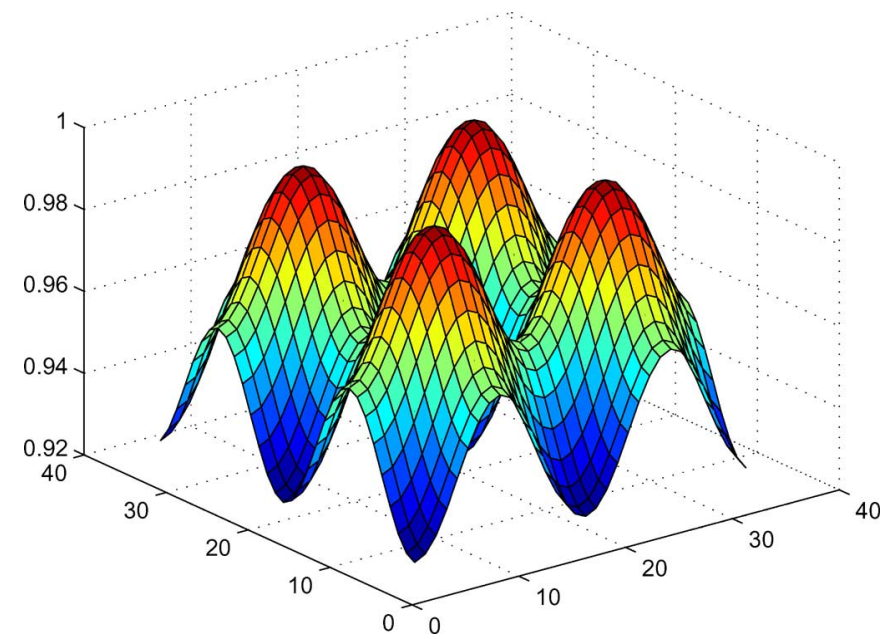

Fig. 3. Two-Dimensional DFT of $\operatorname{det} A$, where $L=2^{9} \cdot 3, \alpha=\beta=2^{5}$ and the windows $g, \gamma$ are Gaussians given by (23). The transform is performed on the group $\mathbb{Z}_{\beta} \times \mathbb{Z}_{\alpha}$, and the axis $x$ and $y$ are the numbers $0-31$, that is representatives of the group $\mathbb{Z}_{\alpha}=\mathbb{Z}_{\beta}$.

\section{CONCLUSION}

In this paper, we described a new method for studying the invertibility of Gabor frame operators. The approach can be applied to both the continuous (on $\mathbb{R}^{d}$ ) and the finite discrete setting, where, in the latter case, we obtained algorithms for directly computing the inverse of Gabor frame-type matrices equivalent to those known in the literature. One of the nice features of this framework is that it allows for a unified approach to the continuous and discrete settings, which is not the case in previous methods. It also leads to a nice matrix-type interpretation of twisted convolutions.

\section{APPENDIX A}

In this Appendix, we are going to describe briefly the proof of Wiener's Lemma. All the details are presented in [5]. What we have to show is that if $A=\phi(a)$, for some $a \in \ell^{1}$, is invertible, then there exists a sequence $b \in \ell^{1}$ such that $A^{-1}=\phi(b)$. The proof is based on the properties of sequences $a^{r, s}$ defined in (13) and Cramer's rule for commutative algebras.

Assume that $A$ is invertible. Then $\operatorname{det} A$ is an invertible sequence in $\left(\ell^{1}\left(\mathbb{Z}^{2}\right), *\right)$. It turns out that it is supported on $\mathbb{Z} \times p \mathbb{Z}$ and so is its inverse $d$. We construct the inverse $C$ of $A$ using Cramer's rule, that is the entries of the inverse matrix are given by

$$
C_{r, s}=(\operatorname{det} A(s, r)) * d \quad s, r=0, \ldots, p-1
$$

where $A(s, r)$ is a new matrix obtained from $A$ by substituting sth row of $A$ with a vector of zeros having $\delta$ on the $r$ th position, and $r$ th column with a column of zeros having $\delta$ on the $s$ th position. For each $r=0, \ldots, p-1, C_{r, 0}$ is a sequence supported on $\mathbb{Z} \times(r+p \mathbb{Z})$; hence, the entries of the first column of $C$ have disjoint supports. We define a sequence $b$ by summing up the entries of the first column of $C$, i.e.,

$$
b=C_{0,0}+C_{1,0}+\cdots+C_{p-1,0}
$$


and create a new matrix $B$ as an image of the sequence $b$ under the mapping $\phi, B=\phi(b)$. That is, $B$ has the same form as $A$. Finally, it can be shown that $B=C$, i.e., $B$ is indeed the inverse of $A$.

\section{APPENDIX B}

Here, we prove Theorem 6.1.

Proof: Let $k, l \in \mathbb{Z}$

$$
\begin{aligned}
\left(\mathcal{F} \psi_{r, s}\right)(k, l) \\
=\int_{0}^{\alpha} \int_{0}^{\beta} \psi_{r, s}(\xi, x) e^{-2 \pi i k x / \alpha} e^{-2 \pi i l \xi / \beta} d x d \xi \\
=\frac{1}{\alpha} \int_{0}^{\alpha} \int_{0}^{\beta} \sum_{m, n \in \mathbb{Z}} a_{m, s-r-p n} e^{-2 \pi i \frac{m r}{\alpha \beta}} \\
\quad \times e^{2 \pi i\left(\frac{(m-k) x}{\alpha}+\frac{(n-l) \xi}{\beta}\right)} d x d \xi \\
=\alpha^{-1} \sum_{m, n \in \mathbb{Z}} a_{m, s-r-p n} e^{-2 \pi i \frac{m r}{\alpha \beta}} \\
\quad \times \int_{0}^{\alpha} e^{2 \pi i \frac{(m-k) x}{\alpha}} d x \times \int_{0}^{\beta} e^{2 \pi i \frac{(n-l) \xi}{\beta}} d \xi \\
=\beta \sum_{m, n \in \mathbb{Z}} a_{m, s-r-p n} e^{-2 \pi i \frac{m r}{\alpha \beta}} \delta_{m, k} \delta_{n, l} \\
=\beta e^{-2 \pi i \frac{k r}{\alpha \beta}} a_{k, s-r-p n} \beta \omega^{k r} a_{k, s-r-p n} .
\end{aligned}
$$

Therefore

$$
\mathcal{F}\left(\psi_{r, s}\right)=\beta \sum_{l=0}^{p-1} \omega^{l r} a^{l, s-r}=\beta A_{s, r} .
$$

Let $k, l \in \mathbb{Z}$ and $S_{p}$ a group of permutations of the set $\{0, \ldots, p-1\}$, then by the definition of the determinant and previous calculations

$$
\begin{aligned}
(\mathcal{F} & \operatorname{det} \Psi)(k, l) \\
& =\left(\mathcal{F} \sum_{\sigma \in S_{p}}(-1)^{\sigma} \psi_{0, \sigma(0)} \psi_{1, \sigma(1)} \cdots \psi_{p-1, \sigma(p-1)}\right)(k, l) \\
& =\left(\sum_{\sigma \in S_{p}}(-1)^{\sigma} \mathcal{F} \psi_{0, \sigma(0)} * \cdots * \mathcal{F} \psi_{p-1, \sigma(p-1)}\right)(k, l) \\
& =\left(\sum_{\sigma \in S_{p}}(-1)^{\sigma} \beta A_{\sigma(0), 0} * \cdots * \beta A_{\sigma(p-1), p-1}\right)(k, l) \\
& =\beta^{p}\left(\sum_{\sigma \in S_{p}}(-1)^{\sigma} A_{\sigma(0), 0} * \cdots * A_{\sigma(p-1), p-1}\right)(k, l) \\
& =\beta^{p}(\operatorname{det} A)(k, l) .
\end{aligned}
$$

\section{APPENDIX C}

Before proving Theorem 6.2, we come back to a matrix-valued function $G$ introduced in Section 5.1 and state its two important properties:

$$
\begin{aligned}
G_{j, l}(x) & =G_{j+p k, l+p k}(x) \\
G_{j, l}(x) & =G_{0, l-j}(x-j / \beta) .
\end{aligned}
$$

The first property is based on the fact that $q \alpha=p / \beta$. The second relation shows that $G_{j, l}(x)$ can be derived from $G_{0, l}(x)$. Next, we define the entries of the $(p \times p)$-matrix valued function $\widehat{G}(\xi, x)$ by

$$
\widehat{G}_{r, s}(\xi, x)=\sum_{k \in \mathbb{Z}} G_{r, s+p k}(x) e^{-2 \pi i k \xi / \beta}, \quad r, s=0, \ldots, p-1 .
$$

As shown in [7, Ch. 13] (replace one $g$ by $\gamma$ ), $\widehat{G}(\xi, x)$ has an absolutely converging Fourier series expansion and is, therefore, continuous and of period $(\beta, \alpha)$. Moreover, $G(x)$ is invertible on $\ell^{2}(\mathbb{Z})$ if and only if the $(p \times p)$-matrix $\widehat{G}(\xi, x)$ is invertible for every $(\xi, x)$.

Lemma 8.1: The matrix-valued function $\Psi(\xi, x)$ defined in (20) coincides with the matrix-valued function $\widehat{G}(\xi, x)$ defined in (26).

Proof: Using (18), (24), and (25), we compute

$$
\begin{aligned}
\widehat{G}_{r, s}(\xi, x) & =\sum_{n \in \mathbb{Z}} G_{r, s+p n}(x) e^{-2 \pi i n \xi / \beta} \\
& =\sum_{n \in \mathbb{Z}} G_{0, s-r+p n}(x-r / \beta) e^{-2 \pi i n \xi / \beta} \\
& =\alpha^{-1} \sum_{m, n \in \mathbb{Z}} a_{m, s-r+p n} e^{\frac{2 \pi i m(x-r / \beta)}{\alpha}} e^{\frac{-2 \pi i n \xi}{\beta}} \\
& =\alpha^{-1} \sum_{m, n \in \mathbb{Z}} a_{m, s-r-p n} e^{\frac{-2 \pi i m r}{\alpha \beta}} e^{2 \pi i\left(\frac{m x}{\alpha}+\frac{n \xi}{\beta}\right)} \\
& =\psi_{r, s}(\xi, x) .
\end{aligned}
$$

Now, we are in the position to prove Theorem 6.2.

Proof: Let $(x, \xi) \in[0, \alpha / p] \times[0,1 / \alpha]$. Then

$$
\begin{aligned}
& \mathcal{A}_{r, s}(x, \xi) \\
& =\alpha \sum_{j=0}^{q-1} \overline{\mathcal{Z}_{\alpha} g(x+\alpha s / p, \xi-\beta j)} \\
& \times \mathcal{Z}_{\alpha} \gamma(x+\alpha r / p, \xi-\beta j) e^{2 \pi i j(r-s) / q} \\
& =\alpha \sum_{m, n \in \mathbb{Z}} \overline{g(x+\alpha s / p-\alpha m)} \gamma(x+\alpha r / p-\alpha n) \\
& \times e^{-2 \pi i \alpha \xi(m-n)} \sum_{j=0}^{q-1} e^{2 \pi i j(r-s+p(m-n)) / q} \\
& =\alpha \sum_{m, k \in \mathbb{Z}} \overline{g(x+\alpha s / p-\alpha m)} \gamma(x+\alpha r / p-\alpha(m-k)) \\
& \times e^{-2 \pi i \alpha \xi k} \sum_{j \in \mathbb{Z}_{q}} e^{2 \pi i j(r-s+p k) / q} \\
& \left.=\alpha q \sum_{m, k \in \mathbb{Z}} \overline{g(x+\alpha s / p-\alpha m)} \gamma(x+\alpha r / p-\alpha m+\alpha k)\right) \\
& \times e^{-2 \pi i \alpha \xi k} \delta_{q \mathbb{Z}, r-s+p k} .
\end{aligned}
$$


Since $\operatorname{gcd}(p, q)=1$, there exist $k_{0}, l_{0} \in \mathbb{Z}$ such that $p k_{0}+$ $q l_{0}=1$. Then $r-s+p k \in q \mathbb{Z}$ if and only if $k \in-k_{0}(r-$ $s)+q \mathbb{Z}$. Therefore, by changing summation over $m$ to $m+k_{0} s$ and noticing that for every $s \in \mathbb{Z}_{p},(\alpha / p) s-\alpha k_{0} s\left(s l_{0} / \beta\right)$, we obtain

$$
\begin{aligned}
\mathcal{A}_{r, s}(x, \xi)= & \alpha q \sum_{m, l \in \mathbb{Z}} \overline{g\left(x+s l_{0} / \beta-\alpha m\right)} \\
& \times \gamma\left(x+\frac{\left(r l_{0}+p l\right)}{\beta}-\alpha m\right) e^{2 \pi i\left(k_{0}(r-s) \alpha \xi-l \frac{p \xi}{\beta}\right)} \\
= & \alpha q \sum_{l \in \mathbb{Z}} G_{-r l_{0}-p l,-s l_{0}}(x) e^{-2 \pi i l \frac{p \xi}{\beta}} e^{2 \pi i k_{0}(r-s) \alpha \xi} \\
= & \alpha q \sum_{l \in \mathbb{Z}} G_{-r l_{0},-s l_{0}+p l}(x) e^{-2 \pi i l \frac{p \xi}{\beta}} e^{2 \pi i k_{0}(r-s) \alpha \xi} \\
= & \alpha q e^{2 \pi i k_{0}(r-s) \alpha \xi} \widehat{G}_{-r l_{0},-s l_{0}}(p \xi, x) .
\end{aligned}
$$

By Lemma 8.1, $\Psi(\xi, x)$ coincides with the matrix-valued function $\widehat{G}(\xi, x)$. Hence

$$
\mathcal{A}_{r, s}(x, \xi)=\alpha q e^{2 \pi i k_{0}(r-s) \alpha \xi} \psi_{-r l_{0},-s l_{0}}(p \xi, x) .
$$

By Cramer's rule

$$
\begin{aligned}
\operatorname{det} \mathcal{A}(x, \xi)= & \sum_{\sigma \in S_{p}}(-1)^{\sigma} \mathcal{A}_{\sigma(0), 0}(x, \xi) \cdots \mathcal{A}_{\sigma(p-1), p-1}(x, \xi) \\
= & (\alpha q)^{p} \sum_{\sigma \in S_{p}}(-1)^{\sigma} \\
& \times \prod_{i \in \mathbb{Z}_{p}} e^{2 \pi i k_{0}(\sigma(i)-i) \alpha \xi} \widehat{G}_{-\sigma(i) l_{0},-i l_{0}}(p \xi, x) \\
= & (\alpha q)^{p} \sum_{\sigma \in S_{p}}(-1)^{\sigma} \underbrace{e^{2 \pi i k_{0} \alpha \xi \sum_{i=0}^{p-1}(\sigma(i)-i)}}_{=1} \\
& \times \prod_{i \in \mathbb{Z}_{p}} \widehat{G}_{-\sigma(i) l_{0},-i l_{0}}(p \xi, x) \\
= & (\alpha q)^{p} \sum_{\tau \in S_{p}}(-1)^{\tau} \widehat{G}_{\tau(0), 0}(p \xi, x) \cdots \\
& \widehat{G}_{\tau(p-1), p-1}(p \xi, x) \\
= & (\alpha q)^{p} \operatorname{det} \widehat{G}(p \xi, x)=(\alpha q)^{p} \operatorname{det} \Psi(p \xi, x)
\end{aligned}
$$

which completes the proof.

The above theorem is also true in higher dimensions with obvious changes in the proof.

\section{ACKNOWLEDGMENT}

The authors would like to thank K. Gröchenig and Y. Y.Zeevi for many fruitful discussions.

\section{REFERENCES}

[1] J. ben Arie, Z. Wang, and K. R. Rao, “Affine invariant shape representation and recognition using Gaussian kernels and multi-dimensional indexing," in Proc. IEEE Int. Conf. Acoustics, Speech, Signal Processing, 1996, pp. 3470-3473.

[2] O. Christensen, An Introduction to Frames and Riesz Bases. Boston, MA: Birkhäuser, 2003.

[3] I. Daubechies, A. Grossmann, and Y. Meyer, "Painless nonorthogonal expansions," J. Math. Phys., vol. 27, no. 5, pp. 1271-1283, 1986.

[4] P. J. Davis, Circulant Matrices, 2nd ed. New York: AMS Chelsea, 1994.
[5] Y. C. Eldar, E. Matusiak, and T. Werther, "Constructive proof of Wiener's lemma for twisted convolution," 2005.

[6] H. G. Feichfcinger and G. Zimmermann, "A Banach space of test functions for Gabor analysis," in Gabor Analysis and Algorithms: Theory and Applications. Boston, MA: Birkhäuser, 1998, pp. 123-170.

[7] K. Gröchenig, Foundations of Time-Frequency Analysis. Boston, MA: Birkhäuser, 2001.

[8] A. K. Jain and F. Farrokhnia, "Unsupervised texture segmentation using Gabor filters," Pattern Recognit., vol. 24, no. 12, pp. 1167-1186, 1991.

[9] A. J. E. M. Janssen, "Duality and biorthogonality for Weyl-Heisenberg frames," J. Fourier Anal. Appl., vol. 1, no. 4, pp. 403-436, 1995.

[10] S. Li, "On general frame decompositions," Numer. Fundam. Anal. Optim., vol. 16, no. 9, pp. 1181-1191, 1995.

[11] B. S. Manjunath and W. Y. Ma, "Texture features for browsing and retrieval of image data," IEEE Trans. Pattern Anal. Mach. Intell., vol. 18, no. 8, pp. 837-842, Aug. 1996.

[12] S. Qiu and H. G. Feichtinger, "Discrete Gabor structures and optimal representation," IEEE Trans. Signal Process., vol. 43, no. 10, pp. 2258-2268, Oct. 1995.

[13] T. Strohmer, "Numerical algorithms for discrete Gabor expansions," in Gabor Analysis and Algorithms: Theory and Applications. Boston, MA: Birkhäuser, 1998, pp. 267-295.

[14] T. Werther, Y. C. Eldar, and N. Subbanna, "Dual Gabor frames: Theory and computational aspects," IEEE Trans. Signal Process., vol. 53, no. 11, pp. 4147-4158, Nov. 2005

[15] P. J. Wolfe, M. Dörfler, and S. J. Godsill, "Multi-Gabor dictionaries for audio time-frequency analysis," in Proc. IEEE Workshop of Signal Processing to Audio and Acoustics, 2001, pp. 43-46.

[16] M. Zibulski and Y. Y. Zeevi, "Analysis of multiwindow Gabor-type schemes by frame methods," Appl. Comput. Harmon. Anal., vol. 4, no. 2, pp. 188-221, 1997.

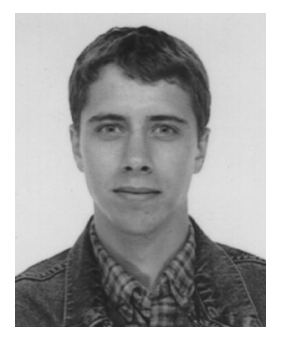

Tobias Werther received the diploma and the Ph.D. degree in mathematics from the University of Vienna, Vienna, Austria, in 2000 and 2003, respectively.

From July 2004 to September 2004, he was a Postdoctoral Fellow with the Department of Electrical Engineering, The Technion-Israel Institute of Technology, Haifa. He is currently a Research Assistant with the Numerical Harmonic Analysis Group, Faculty of Mathematics, University of Vienna, under the direction of Prof. H. G. Feichtinger. His current research areas include harmonic analysis and spline

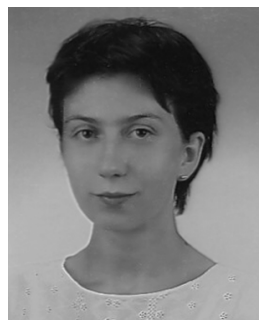

Ewa Matusiak received the interdisciplinary M.S degree in mathematics and computer engineering from the University of Oklahoma, Norman, in 2003. She is currently pursuing the Ph.D. degree in mathematics at the University of Vienna, Vienna, Austria under the direction of Prof. H. G. Feichtinger.

From July 2005 to September 2005, she was a graduate Fellow at the Department of Electrical Engineering, The Technion-Israel Institute of Technology, Haifa. Her research interests are time-frequency analysis and uncertainty principles.

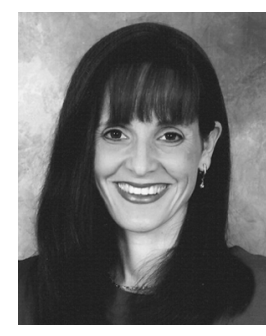

Yonina C. Eldar (S'98-M'02) received the B.Sc. degree in physics and the B.Sc. degree in electrical engineering from Tel-Aviv University (TAU), Tel-Aviv, Israel, in 1995 and 1996, respectively, and the Ph.D. degree in electrical engineering and computer science from the Massachusetts Institute of Technology (MIT), Cambridge, in 2001

From January 2002 to July 2002, she was a Postdoctoral Fellow at the Digital Signal Processing Group, MIT. She is currently a Senior Lecturer with the Department of Electrical Engineering, The Technion-Israel Institute of Technology, Haifa, Israel. She is also a Research 
Affiliate with the Research Laboratory of Electronics, MIT. She serves on the Signal Processing Theory and Methods Technical Committee. Her current research interests are in the general areas of signal processing, statistical signal processing, and quantum information theory.

Dr. Eldar was in the program for outstanding students at TAU from 1992 to 1996. In 1998, she held the Rosenblith Fellowship for study in Electrical Engineering at MIT, and, in 2000, she held an IBM Research Fellowship. In 2005, she received the Wolfe Foundation Krill Prize. She is currently a Horev Fellow of the Leaders in Science and Technology program at the Technion as well as an Alon Fellow.

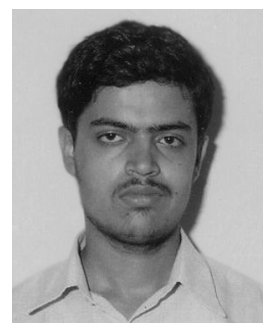

Nagesh K. Subbanna was born in 1978. He received the B.E. degree in computer science (with first class honors) from Bangalore University, Bangalore, India, in 2001. He is currently pursuing the M.Sc. degree in electrical engineering at The Technion-Israel Institute of Technology, Haifa.

$\mathrm{He}$ was with the Computer Vision Laboratory, Electrical Engineering Department, IISc, India, for one year. His research interests include time-frequency analysis, image processing, and bioinformatics. 